\title{
Paternal Programming of Liver Function and Lipid Profile Induced by a Paternal Pre-Conceptional Unhealthy Diet: Potential Association with Altered Gut Microbiome Composition
}

\author{
Xiaoli Zhang a, b Yunpeng Dong ${ }^{c}$ Guoying Sun ${ }^{a}$ Ahmed A. Hasan ${ }^{\text {b, d }}$ \\ Mei Tian ${ }^{a}$ e Suimin Zeng ${ }^{\text {a, e }}$ Jing Li $^{a}$ Shiyu Zeng ${ }^{a}$ Jun Ma ${ }^{a}$ Jian Lia, e \\ Berthold Hocher ${ }^{\text {b, }}$ - $-g$ \\ a'Department of Basic Medicine, Hunan Normal University, School of Medicine, Changsha, \\ China; ${ }^{b}$ Fifth Department of Medicine (Nephrology/Endocrinology/Rheumatology), \\ University Medical Centre Mannheim, University of Heidelberg, Mannheim, Germany; \\ 'Department of Otolaryngology Head and Neck Surgery, Institute of Otology, the Second \\ Xiangya Hospital, Central South University, Changsha, China; ${ }^{2}$ Department of Biochemistry, \\ Faculty of Pharmacy, Zagazig University, Zagazig, Egypt; ${ }^{\mathrm{e}}$ Key Laboratory of Study and \\ Discovery of Small Targeted Molecules of Hunan Province, School of Medicine, Hunan \\ Normal University, Changsha, China; ${ }^{f}$ LADR GmbH, MVZ Neuruppin, Neuruppin, Germany; \\ gReproductive and Genetic Hospital of CITIC-Xiangya, Changsha, China
}

\section{Keywords}

Gut microbiota $\cdot$ Liver function · High-fat-sucrose-salt diet $\cdot$ Paternal programing

\begin{abstract}
Background/Aims: Paternal exposure to adverse environmental conditions can act on offspring's phenotype and influence offspring's later life disease risk. Our study was designed to examine the effect of feeding male rats before mating a high-fat, high-sucrose and high-salt diet (HFSSD) over two generations (F0 and F1) on their offspring's (F2) liver function and gut microbiome composition. Methods: Male F0 rats and male F1 rats were fed either control diet or HFSSD before mating. Liver function of F2 offspring was investigated, and their gut microbiome composition was analyzed by 16S rRNA gene sequencing in the F2 offspring of rats whose fathers and grandfathers were fed with control diet (CD) (FOCD+F1CD-F2 group) or HFSSD prior to mating (FOHD+F1HD-F2 group). Results: F2 offspring had higher serum as-
\end{abstract}

X.Z., Y.D. and G.S. contributed equally to this work.

Prof. Dr. Berthold Hocher

Fifth Department of Medicine, University Medical Centre Mannheim University of Heidelberg

DE-68167 Mannheim (Germany)

E-Mail berthold.hocher @ medma.uni-heidelberg.de
Dr. Jian Li

Department of Basic Medicine

Hunan Normal University, School of Medicine

Changsha 410013 (China)

E-Mail angela13656355@qq.com 


\section{Kidney \\ Blood Pressure \\ Research}

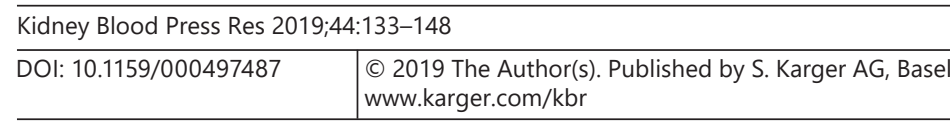

Zhang et al.: Gut Microbiome and Paternal Programming

partate aminotransferase activity (female, $p<0.05$ and male, $p<0.01$ respectively) compared with control. Shannon indexes of gut microbiota indicated a significantly higher diversity in the female F0HD+F1HD-F2 as compared to F0CD+F1CD-F2 female offspring $(p<0.01)$. The dominant phyla of all the groups were Bacteroidetes, Firmicutes and Proteobacteria. There were significant differences in gut bacterial community composition at phyla and genus level between the FOCD+F1CD-F2 and FOHD+F1HD-F2. Furthermore, the variation in the relative abundance (percentage) of bacterial genus in the $\mathrm{F} 2$ offspring was associated with liver function alterations induced by a paternal pre-conceptional unhealthy diet. Male FOHD+F1HD-F2 offspring had higher serum cholesterol, high density lipoproteins as well as low density lipoproteins concentrations compared to the corresponding male control rats. Conclusion: Taken together, our findings suggested that a paternal pre-conceptional unhealthy diet predisposes the offspring to mild liver function alterations and alterations of gut microbiota in later life. Effects on lipids were sex-specific and only seen in male offspring.

(C) 2019 The Author(s)

Published by S. Karger AG, Basel

\section{Introduction}

Disadvantageous nutrition of the parents can impact on later life disease susceptibility of the offspring. Suboptimal early life conditions that affect germ cells, the intrauterine environment or early postnatal conditions may be responsible for this. The concept connecting the impact of early life conditions on later life disease susceptibility is known as fetal programming or developmental origins of adult disease [1-3].

Recently, gut microbiota, which plays important roles in maintaining host health and in disease development, has become a major and promising research area in biomedicine [4-7]. Previous studies have suggested that gut microbiota is important in regulating metabolic pathways in healthy people and in patients with obesity, diabetes and cardiovascular diseases $[8,9]$. The developmental origins of health and disease (DOHaD) hypothesis, firstly formulated in the early 1990s, proposed that adverse in utero conditions can influence developmental pathways in early life that result in long-term changes to offspring disease susceptibility $[1,2,10,11]$. Recent work on the human microbiome indicates that gut microbiota may additionally explain the observations put forth by the DOHaD hypothesis [12-14].

Alterations in maternal microbiota composition, associated with both diet and obesity status, have been widely reported [15-18]. Disruption of the mother's gut microbiota can influence early pioneering bacteria in the newborn and may be a prime candidate for intergenerational transmission of metabolic disease risk given the considerable influence of these microbes on infant growth $[19,20]$, immune system development [21-23], and even neural development $[24,25]$. Interestingly, emerging evidence has additionally indicated that transmission of microbiota from mother to offspring may occur before delivery, which has since reemphasized the importance of the pregnancy period to the development of the neonatal microbiome $[12,13]$. Gut microbiota is an important factor that affects energy harvest from the diet and energy storage in the host. Indeed, gut microbiota exerts a strong influence on host lipid and cholesterol metabolism. It was reported that the severity of nonalcoholic fatty liver disease (NAFLD) postnatally is strongly associated with gut dysbiosis and a shift in gut metabolic function [26], including production and utilization of short-chain fatty acids and bile acids.

The above-described mechanisms are of maternal origin and are caused by either maternal environmental factors or maternal genes that act on the epigenome of the growing fetus. Several studies showed that also paternal nutritional factors, in particular during maturation of the sperm, may alter the epigenome and the phenotype of the offspring [27-30]. 
Table 1. Composition of $\mathrm{CD}$, and HFSSD

\begin{tabular}{lcc}
\hline & CD & HFSSD \\
\hline Components & & \\
Casein & 20.0 & 23.3 \\
Corn starch & 39.8 & 8.7 \\
Maltodextrin & 13.2 & 10.5 \\
Sucrose & 10.0 & 20.1 \\
Fiber & 5.0 & 5.0 \\
Soybean oil & 7.0 & 2.9 \\
Lard & 0.0 & 20.7 \\
Salt & 0.3 & 3.7 \\
Minerals & 3.5 & 3.5 \\
Vitamins & $1.0(0.01$ folate $)$ & $1.0(0.01$ folate $)$ \\
\hline Energy & & \\
Energy value, kJ/kg & 16,736 & 19,539 \\
Lipids & 15.8 & 45.5 \\
Sucrose & 10.00 & 17.25 \\
Proteins & 20.30 & 20.23 \\
\hline
\end{tabular}

All values are given as percentages unless indicated otherwise. All components are wt/wt. CD, control diet; HFSSD, high-fat, high-sucrose and high-salt diet.

It was shown that feeding of male rat founders with high-fat diet before mating induces impaired glucose tolerance in female offspring [31], most likely due to epigenetic adaptations in liver. However, investigations into the later-life effects of paternal pre-conceptional unhealthy diet on gut microbiota in the offspring are limited. Given the multifaceted nature of this topic, in the current study, we analyzed the effect of feeding male rats high-fat, highsucrose and high-salt diet (HFSSD) over two generations (F0 and F1) on their offspring's (F2) liver function and gut microbiome composition.

\section{Materials and Methods}

\section{Animals}

The present study utilized Sprague-Dawley rats of both sexes, including F0 generation (30 males and 32 females), F1 generation ( 80 males and 80 females) and F2 generation ( 49 males and 38 females) animals. The F0 rats were purchased from Hunan SJA Laboratory Animal Co., Ltd (Changsha, China) and were delivered at the age of 4 weeks. The animals were housed in temperature-controlled chambers under control lighting with 12:12-h light-dark cycles. All the animals were allowed ad libitum access to water and food. The experimental protocols were approved by the Experimental Animal Center of the Hunan Normal University (Changsha, China) in accordance with the ethical standards of Hunan Province, China.

\section{Study Design}

F0 male rats were randomly divided into two study groups: (1) control diet (CD, $n=15$ ) and (2) HFSSD $(n=15)$. All animals were allowed to consume tap water and food ad libitum. A detailed description of the diet compositions is indicated in Table 1.

The 14-week-old F0 founder males fed either a CD or HFSSD were mated with F0 12-week-old normal weight naturally cycling CD-fed dams to produce F1 offspring. The presence of a vaginal plug was designated as gestational day 1 . The rats were mated for 4 days. After mating and throughout the gestational period, all the F1 dams were fed CD. The F1 male offspring was randomly allocated into two study groups. (1) Paternal control diet group (PatCD group): male F1 offspring of CD-fed F0 founders received CD from birth until the 24 th week of age and were mated with CD-fed dams and their F2 offspring represented the F0CD+F1CD 


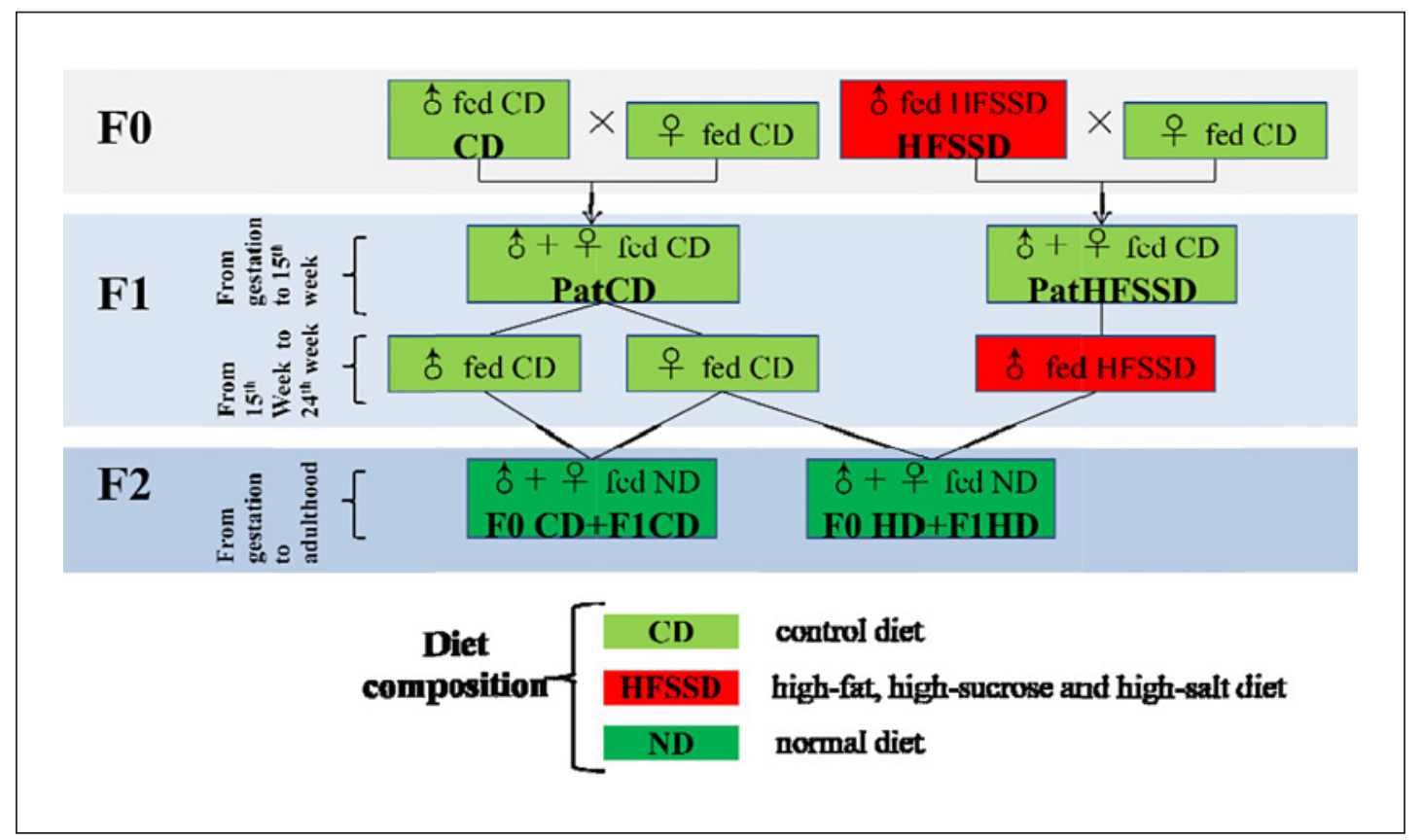

Fig. 1. F0 male rats were randomly divided into two study groups: (1) control diet (CD, $n=15)$; (2) high-fat, high-sucrose and high-salt diet (HFSSD, $n=15$ ). The F1 male offspring was randomly allocated to two study groups: (1) paternal control diet group (PatCD group): male F1 offspring of CD-fed F0 founders received CD from birth until the 24th week of age and were mated with CD-fed dams and their F2 offspring represented the F0CD+F1CD group (F0CD+F1CD-F2 group); (2) paternal high-fat, high-sucrose and high-salt diet group (PatHFSSD group): male F1 offspring of HFSSD-fed F0 founders received CD from birth until the 15th week of age followed by HFSSD until the 24th week of age and were mated with CD-fed dams and their offspring represented the F0HD+F1HD group (F0HD+F1HD-F2 group). Based on F0 and F1 paternal diet before mating, the F2 offspring can be divided into two study groups: (1) F0CD+F1CD group: F2 offspring of F0 and F1 male founders fed a CD; (2) F0HD+F1HD group (F0CD+F1CD-F2 group): F2 offspring of F0 and F1 male founders fed a HFSSD (F0HD+F1HD-F2 group).

group (F0CD+F1CD-F2 group); (2) paternal high-fat, high-sucrose and high-salt diet group (PatHFSSD group): male F1 offspring of HFSSD-fed F0 founders received CD from birth until the 15th week of age followed by HFSSD until the 24th week of age and were mated with CD-fed dams and their offspring represented F0HD+F1HD group (F0HD+F1HD-F2 group). In summary, based on F0 paternal diet and F1 paternal diet before mating, the F2 offspring can be divided into two study groups (Fig. 1): (1) F0CD+F1CD group: F2 offspring of F0 and F1 male founders fed a CD; (2) F0HD+F1HD group: F2 offspring of F0 and F1 male founders fed a HFSSD. All F2 offspring (F0CD+F1CD and F0HD+F1HD) were fed normal diet (ND) from birth until the 25 th week of age. Then, they were sacrificed under deep anesthesia induced by intraperitoneal injection of $3 \%$ (wt/vol.) sodium pentobarbital solution. Afterwards, blood samples were collected, the organs were harvested, and feces were aseptically taken from the cecum of the rats.

\section{Metabolic Tests}

Blood samples were taken via aortic puncture at study end. Serum glucose (GLU), cholesterol (CHOL), triacylglycerols (TG), high-density lipoproteins (HDL), low-density lipoproteins (LDL), alanine aminotransferase (ALT), and aspartate transaminase (AST) were measured using Hitachi 7020 automatic biochemistry analyzer (Hitachi High-Technologies, Tokyo, Japan).

\section{Fecal DNA Extraction and 16 S rRNA Gene Sequencing}

Fresh stool samples were collected after sacrifice and immediately stored at $-80^{\circ} \mathrm{C}$ for subsequent analysis. The sample sizes were 25 (16 male samples and 9 female samples) in F0CD+F1CD (F2 offspring of F0 and F1 male founders fed a CD) group and 29 (16 male samples and 13 female samples) in F0HD+F1HD 
Table 2. Body weight and serum metabolite levels in F0 founders males

\begin{tabular}{lcc}
\hline Parameter & CD $(n=15)$ & HFSSD $(n=15)$ \\
\hline Body weight, $g$ & & \\
On arrival in the lab & $175.5 \pm 2.9$ & $177.2 \pm 5.2$ \\
At 10 weeks & $415.5 \pm 7.3$ & $396.1 \pm 4.9$ \\
At 12 weeks & $472.4 \pm 7.5$ & $447.1 \pm 4.1^{* *}$ \\
At 14 weeks & $504.4 \pm 8.1$ & $475.8 \pm 4.8^{* *}$ \\
At 18 weeks & $562.6 \pm 8.4$ & $539.6 \pm 4.1^{*}$ \\
\hline Serum metabolites & & \\
GLU, mmol/L & $7.6 \pm 1.5$ & $7.6 \pm 1.7$ \\
TG, mmol/L & $1.1 \pm 0.1$ & $1.0 \pm 0.1$ \\
CHOL, mmol/L & $1.4 \pm 0.1$ & $1.7 \pm 0.1^{*}$ \\
BUN, mmol/L & $7.0 \pm 0.3$ & $8.3 \pm 0.3^{* *}$ \\
Cr, $\mu$ mol/L & $35.3 \pm 2.2$ & $35.4 \pm 2.1$ \\
AST, U/L & $144.3 \pm 14.2$ & $142.5 \pm 9.6$ \\
ALT, U/L & $26.6 \pm 6.3$ & $59.8 \pm 16.2^{*}$ \\
\hline
\end{tabular}

GLU, glucose; TG, triacylglycerols; CHOL, cholesterol; BUN, blood urea nitrogen; Cr, creatinine; AST, aspartate aminotransferase; ALT, alanine aminotransferase; CD, control diet; HFSSD, high-fat, highsucrose and high-salt diet. Values are given as mean \pm SEM. ${ }^{*} p<0.05$ versus $\mathrm{CD},{ }^{* *} p<0.01$ versus CD.

(F2 offspring of F0 and F1 male founders fed a HFSSD) group. Genomic DNA of microbiota was extracted from fecal samples by QIAamp DNA Stool Mini Kit (Qiagen, Hilden, Germany) according to the manufacturer's instructions. DNA was quantified by the Nanodrop 2000. The extracted DNA from each sample was used as the template to amplify the V3 and V4 hypervariable regions of ribosomal 16S rRNA genes. Briefly, the purified $1 \mu \mathrm{g}$ of genomic DNA was fragmented to an average size of 300-400 bp and ligated with adapters. The PCR was performed using a primer cocktail that anneals to the ends of the adapters to enrich DNA fragments that have adapter molecules on both ends and followed by clean up and quantification. Sequencing was performed using a 300-bp paired-end sequencing protocol on the Illumina MiSeqplatform (Illumina, San Diego, CA, USA) at Oebiotech Company, Shanghai, China. Raw paired-end reads were subjected to quality filtering using Trimmomatic software before paired-end read assembling with FLASH software. All chimeras of assembling sequences were eliminated to reach high-quality sequences.

\section{Bioinformatics and Statistical Analysis}

The optimized sequences were clustered into Operational Taxonomic Units (OTUs) using Vsearch software with $97 \%$ threshold of pairwise identity, and the OTUs were used to estimate community diversity and richness. The Shannon index, PD_whole_tree, and Chao were calculated using QIIME. The representative sequences of OTUs were used to generate a phylogenetic tree using FasTree. The phylogenetic tree was then used for unweighted UniFrac principal coordinate analysis (PCoA) [32]. The relative abundances of gut microbiota in each sample and other measurement data were expressed as mean \pm SEM and evaluated with unpaired $t$-test. Correlation analyses between relative abundance (percentage) of bacterial genus and liver function index were performed by using Spearman's correlation analyses. Statistical significance was accepted as $p<0.05$ (two-sided significance testing). All statistical analyses were performed using SPSS 22.0 statistical software (Chicago, IL, USA).

\section{Results}

FO Founders

Male F0 founders were fed either CD or HFSSD for 9 weeks before mating. The body weight gain and serum metabolite levels of the F0 founders are presented in Table 2. No effect of HFSSD on the number of pubs was observed. 
Table 3. Body weight, liver weight and serum metabolites of female F2 offspring

\begin{tabular}{lcc}
\hline Parameter & $\begin{array}{l}\text { F0CD } \\
(n=11)\end{array}$ & $\begin{array}{l}\text { F0HD+F1HD } \\
(n=15)\end{array}$ \\
\hline Body weight, $g$ & & \\
At 9 weeks & $214.35 \pm 4.79$ & $232.36 \pm 3.56^{* *}$ \\
At 12 weeks & $241.20 \pm 5.51$ & $254.17 \pm 3.32$ \\
At 15 weeks & $262.96 \pm 5.39$ & $279.87 \pm 3.77^{*}$ \\
At 18 weeks & $278.07 \pm 4.95$ & $301.32 \pm 3.75^{* *}$ \\
At 22 weeks & $298.15 \pm 5.34$ & $316.65 \pm 3.88^{*}$ \\
At 25 weeks & $308.1 \pm 5.55$ & $322.64 \pm 3.71^{*}$ \\
\hline Liver weight & & \\
Liver weight, g & $9.78 \pm 0.22$ & $10.22 \pm 0.18$ \\
Relative liver weight, & & \\
\% to body weight & $3.08 \pm 0.01$ & $3.13 \pm 0.01$ \\
\hline Serum metabolites & & \\
AST, U/L & $88.75 \pm 6.55$ & $112.10 \pm 7.05^{*}$ \\
ALT, U/L & $33.03 \pm 4.37$ & $40.72 \pm 2.62$ \\
GLU, mmol/L & $7.99 \pm 0.24$ & $8.03 \pm 0.45$ \\
TG, mmol/L & $2.00 \pm 0.31$ & $1.77 \pm 0.18$ \\
CHOL, mmol/L & $2.17 \pm 0.12$ & $2.33 \pm 0.10$ \\
HDL, mmol/L & $0.82 \pm 0.04$ & $0.91 \pm 0.05$ \\
LDL, mmol/L & $0.13 \pm 0.01$ & $0.12 \pm 0.01$ \\
\hline
\end{tabular}

AST, aspartate aminotransferase; ALT, alanine aminotransferase; GLU, glucose; TG, triacylglycerols; CHOL, cholesterol; HDL, high density lipoproteins; LDL, low density lipoproteins; F0CD+F1CD, F2 offspring of F0 and F1 male founders fed a CD; F0HD+F1HD, F2 offspring of F0 and F1 male founders fed a HFSSD. Values are given as mean \pm SEM. ${ }^{*} p$ $<0.05$ versus F0CD+F1CD, ${ }^{* *} p<0.01$ versus F0CD+F1CD.

\section{F2 Offspring}

Body Weight and Food Intake

Compared with the F0CD+F1CD group, the body weight of the female F0HD+F1HD group increased significantly at the 9th, 15th, 18th, 22nd and 25th week (Table 3). However, no significant differences in body weight, except at the age of 12 weeks, were observed between the F0CD+F1CD and F0HD+F1HD male offspring (Table 4). No significant differences in food and water consumption were observed among the groups throughout the experiment (data not shown).

Liver function and Serum Metabolites

There were no significant differences in liver weights and serum ALT activity between the F0CD+F1CD and F0HD+F1HD of both sexes. However, AST activity was significantly higher in female and male F0HD+F1HD groups than comparable F0CD+F1CD groups (Tables $3,4)$.

No significant differences in other serum metabolites were observed between the female F0CD+F1CD group and the female F0HD+F1HD group (Table 3). However, serum CHOL, HDL and LDL were significantly higher in the male F0HD+F1HD group than in the male F0CD+F1CD group (Table 4). The above results indicate that the grandfather's HFSSD and paternal HFSSD resulted in liver function and lipid metabolism abnormalities of F2 offspring.

Analysis of the Diversity and Richness of the Microbiome using 16S rRNA Sequencing

High-throughput sequencing of the bacterial 16S rRNA gene was performed in fecal samples of F0CD+F1CD and F0HD+F1HD groups at the age of 25 weeks to characterize their 
Table 4. Body weight, liver weight and serum metabolites of male F2 offspring

\begin{tabular}{lcc}
\hline Parameter & $\begin{array}{l}\text { F0CD }+\mathrm{F} 1 \mathrm{CD} \\
(n=20)\end{array}$ & $\begin{array}{l}\text { F0HD+F1HD } \\
(n=19)\end{array}$ \\
\hline Body weight, & & \\
At 9 weeks & $353.39 \pm 4.26$ & $356.22 \pm 4.10$ \\
At 12 weeks & $402.52 \pm 7.97$ & $427.02 \pm 5.84^{*}$ \\
At 15 weeks & $465.66 \pm 5.91$ & $461.43 \pm 10.86$ \\
At 18 weeks & $520.89 \pm 6.97$ & $527.28 \pm 8.97$ \\
At 22 weeks & $565.74 \pm 7.91$ & $584.77 \pm 7.44$ \\
At 25 weeks & $585.78 \pm 7.98$ & $604.77 \pm 8.50$ \\
\hline Liver weight & & \\
Liver weight, g & $19.55 \pm 0.57$ & $19.98 \pm 0.33$ \\
Relative liver weight, & & \\
\% to body weigh & $3.34 \pm 0.01$ & $3.27 \pm 0.01$ \\
\hline Serum metabolites & & \\
AST, U/L & $84.66 \pm 3.39$ & $102.15 \pm 4.80^{* *}$ \\
ALT, U/L & $32.07 \pm 1.62$ & $35.49 \pm 2.01$ \\
GLU, mmol/L & $11.89 \pm 0.66$ & $12.04 \pm 0.60$ \\
TG, mmol/L & $1.92 \pm 0.25$ & $1.96 \pm 0.12$ \\
CHOL, mmol/L & $1.80 \pm 0.07$ & $2.01 \pm 0.06^{*}$ \\
HDL, mmol/L & $0.69 \pm 0.03$ & $0.79 \pm 0.03^{* *}$ \\
LDL, mmol/L & $0.10 \pm 0.01$ & $0.11 \pm 0.01^{*}$ \\
\hline
\end{tabular}

AST, aspartate aminotransferase; ALT, alanine aminotransferase; GLU, glucose; TG, triacylglycerols; CHOL, cholesterol; HDL, high density lipoproteins; LDL, low density lipoproteins; F0CD+F1CD, F2 offspring of F0 and F1 male founders fed a CD; F0HD+F1HD, F2 offspring of F0 and F1 male founders fed a HFSSD. Values are given as mean $\pm \mathrm{SEM}$. $* p<0.05$ versus $\mathrm{F} 0 \mathrm{CD}+\mathrm{F} 1 \mathrm{CD},{ }^{* *} p<0.01$ versus F0CD+F1CD.

Table 5. Bacterial diversity analyzed by high-throughput sequencing

\begin{tabular}{|c|c|c|c|c|}
\hline \multirow[t]{2}{*}{ Estimator } & \multicolumn{2}{|l|}{ Female } & \multicolumn{2}{|l|}{ Male } \\
\hline & $\begin{array}{l}\text { F0CD+F1CD } \\
(n=9)\end{array}$ & $\begin{array}{l}\text { F0HD+F1HD } \\
(n=13)\end{array}$ & $\begin{array}{l}\text { F0CD+F1CD } \\
(n=16)\end{array}$ & $\begin{array}{l}\text { F0HD+F1HD } \\
(n=16)\end{array}$ \\
\hline OTUs & $649.00 \pm 30.19$ & $543.08 \pm 28.91$ & $652.50 \pm 39.51$ & $728.38 \pm 21.81$ \\
\hline PD_whole_tree & $24.86 \pm 1.15$ & $20.15 \pm 0.99$ & $23.84 \pm 1.28$ & $26.68 \pm 0.79$ \\
\hline Chao & $840.85 \pm 48.15$ & $683.68 \pm 43.82$ & $837.24 \pm 48.87$ & $909.19 \pm 22.58^{* *}$ \\
\hline Shannon & $5.65 \pm 0.38$ & $6.43 \pm 0.10^{* *}$ & $6.44 \pm 0.18$ & $6.59 \pm 0.11$ \\
\hline
\end{tabular}

OTUs, operational taxonomic units. The number of OTUs, diversity estimator PD_whole_tree, Chao and Shannon were calculated at 3\% distance. F0CD+F1CD, F2 offspring of F0 and F1 male founders fed a CD; F0HD+F1HD, F2 offspring of F0 and F1 male founders fed a HFSSD. Values are given as mean \pm SEM. ${ }^{*} p<$ 0.05 versus $\mathrm{F} 0 \mathrm{CD}+\mathrm{F} 1 \mathrm{CD}, * * * 0.01$ versus $\mathrm{F} 0 \mathrm{CD}+\mathrm{F} 1 \mathrm{CD}$.

gut microbiome. A total of 2,095,124 high-quality sequences were produced in the present study, with an average of 38,798 sequences per sample. The Good's coverage of each group was over $97 \%$, indicating that the $16 \mathrm{~S}$ rDNA sequences identified in these groups represent the majority of bacteria present in the samples of the present study. Community diversity was estimated using the PD_whole_tree, Chao, and Shannon index, and richness was evaluated based on the number of OTUs. The Shannon index was higher in the female F0HD+F1HD group than in the female F0CD+F1CD group (Table 5). The Chao index was higher in the male 

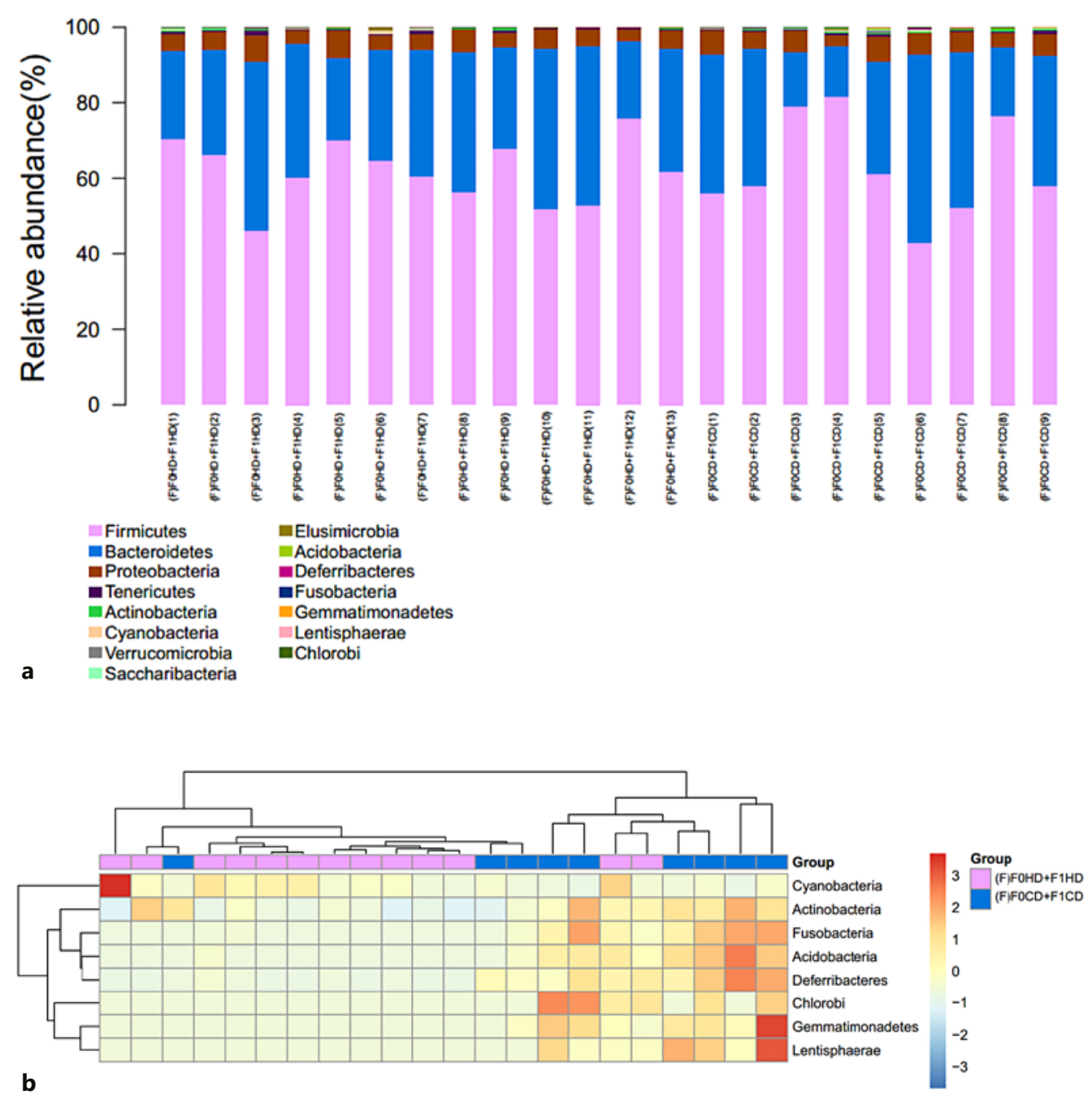

Fig. 2. The gut microbiota composition in F2 generation female offspring. a Relative abundance of the main phyla in the intestinal microbiota. b Heat map showing different phylotypes at the phyla level. $\mathbf{c}$ The heat map reflects the bacterial abundances at the genus level. d Principal coordinate analysis (PCoA) plots of gut microbial communities in F2 generation female offspring.

(Figure continued on next page.)

F0HD+F1HD offspring as compared to female offspring (Table 5). However, no significant differences in the OTUs or PD_whole_tree were observed between the F0CD+F1CD and F0HD+F1HD groups of both sexes (Table 5).

Comparison of the Gut Bacterial Community Composition among Different Groups

We analyzed the abundance of various bacteria in fecal samples. At the phyla level, Firmicutes, Bacteroidetes and Proteobacteria were dominant in the F2 offspring of both sexes (Fig. 2a, 3a). There was no difference in the relative abundances of the three main phyla between the F0CD+F1CD and F0HD+F1HD group of both sexes (Table 6). However, Fusobacteria, Deferribacteres, Gemmatimonadetes, Acidobacteria, Lentisphaerae, Actinobacteria and Chlorobi were significantly decreased whereas Cyanobacteria was significantly increased in 


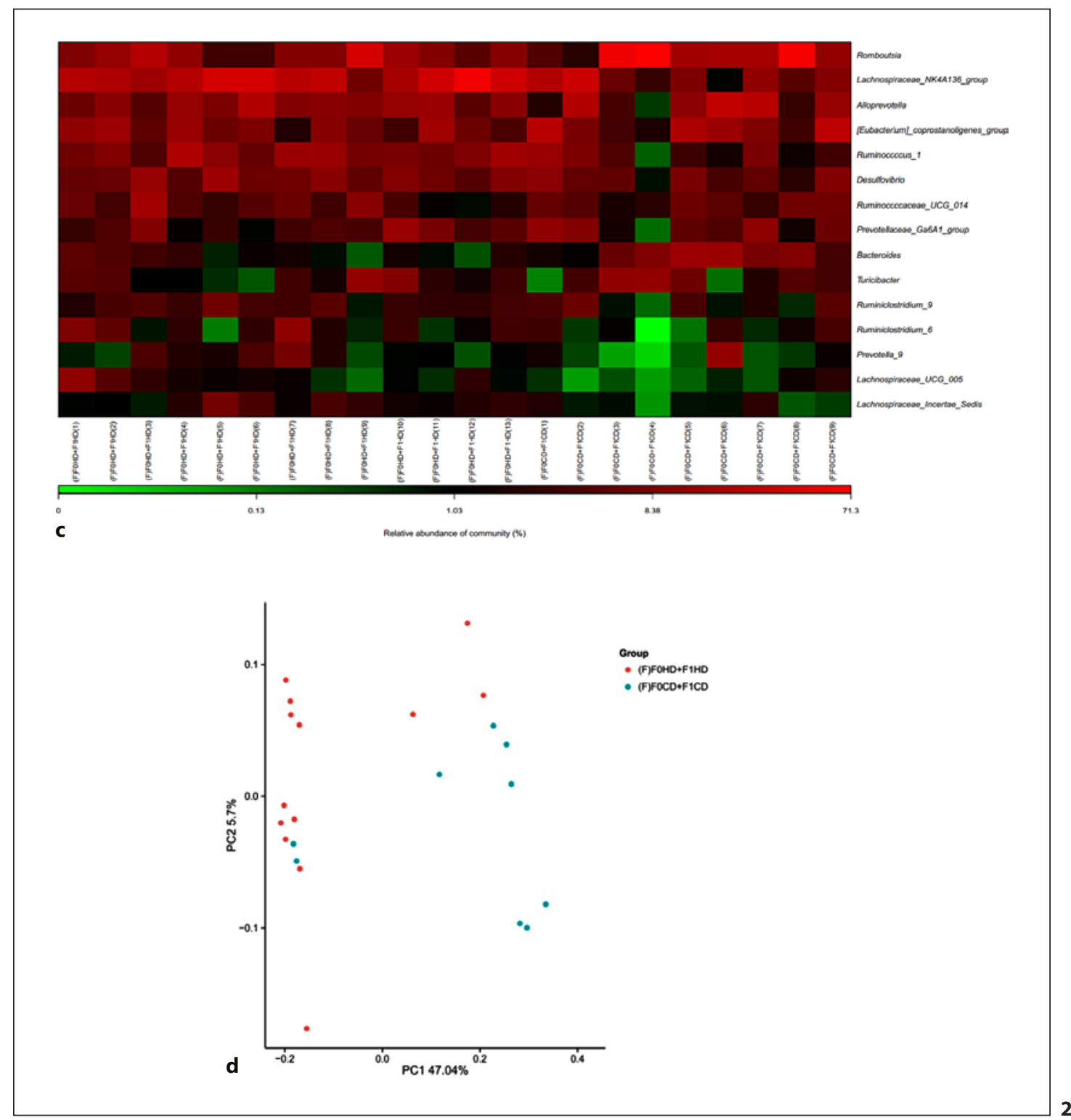

the female F0HD+F1HD group $(p<0.05)$ compared to the female F0CD+F1CD group (Fig. $2 \mathrm{~b})$. A significant increase in the abundance of Gemmatimonadetes and Lentisphaerae was observed in the male F0HD+F1HD group ( $p<0.05$ ) compared to the male F0CD+F1CD group (Fig. 3b).

At the genus level, we analyzed the abundance of the genera in the studied groups and presented the results as a heat map. The resulted heatmap showed that paternal HFSSD led to a lower proportion of Romboutsia (Firmicutes), [Eubacterium]_coprostanoligenes_group (Firmicutes), Bacteroides (Bacteroidetes), Alloprevotella (Bacteroidetes) in F2 female offspring $(p<0.05)$, and a higher proportion of Alloprevotella (Bacteroidetes), Ruminococcaceae UCG_014 (Firmicutes) and Ruminococcus_1 (Firmicutes) $(p<0.05)$ and a lower proportion of Desulfovibrio (Proteobacteria) in F2 male offspring ( $p<0.05$ ) (Fig. 2c, 3c).

Unweighted UniFrac PCoA of Illumina MiSeq amplicon data demonstrated separate clustering of the gut communities between the F0CD+F1CD group and the F0HD+F1HD group with PC1 percent variation explained $=47.04 \%$ and PC2 percent variation explained $=5.7 \%$ in the F2 female offspring (Fig. 2d), and PC1 percent variation explained $=27.73 \%$ and PC2 


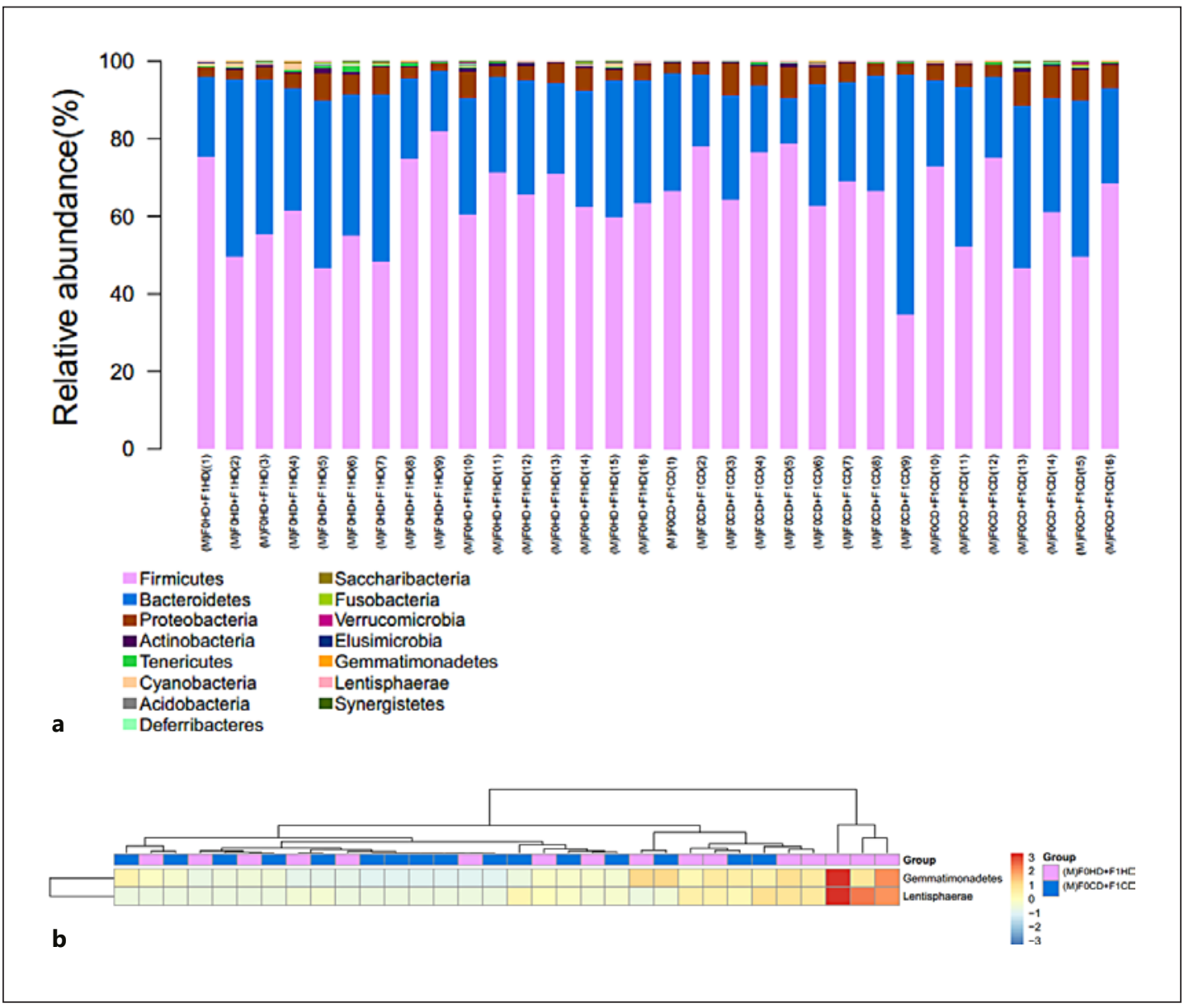

Fig. 3. The gut microbiota composition in F2 generation male offspring. a Relative abundance of the main phyla in the intestinal microbiota. b Heat map showing different phylotypes at the phyla level. c The heat map reflects the bacterial abundances at the genus level. $\mathbf{d}$ Principal coordinate analysis (PCoA) plots of gut microbial communities in F2 generation male offspring.

(Figure continued on next page.)

percent variation explained $=8.58 \%$ in the $\mathrm{F} 2$ male offspring (Fig. $3 \mathrm{~d}$ ). $\mathrm{PCoA}$ revealed striking shifts in microbiota between the F0CD+F1CD group and the F0HD+F1HD group in the F2 offspring, which was mainly reflected on the PC1 axis.

\section{Correlation Analysis}

Spearman's correlation analyses between relative abundance (percentage) of sequences belonging to main bacterial genus and liver function were performed. In the F2 female offspring, the correlation analyses indicated that Lachnospiraceae_NK4A136_group (Firmicutes) $\left(r^{2}=0.446, p<0.05\right)$ and Ruminiclostridium_9 (Firmicutes) $\left(r^{2}=0.525, p<0.05\right)$ percentage were positively associated with AST activity whereas Romboutsia (Firmicutes) $\left(r^{2}=-0.510, p<0.05\right)$ and Bacteroides (Bacteroidetes) $\left(r^{2}=-0.423, p<0.05\right)$ percentage were negatively associated with AST activity. The abundance of Lachnospiraceae_NK4A136_group (Firmicutes) $\left(r^{2}=0.553, p<0.01\right)$, Desulfovibrio (Proteobacteria) $\left(r^{2}=0.441, p<0.05\right)$ and Ruminiclostridium_9 (Firmicutes) ( $\left.r^{2}=0.616, p<0.01\right)$ were positively associated with ALT activity whereas Romboutsia (Firmicutes) $\left(r^{2}=-0.649, p<0.01\right)$ and Bacteroides (Bacteroidetes) $\left(r^{2}=-0.584, p<0.01\right)$ were negatively associated with ALT activity. The abundance 


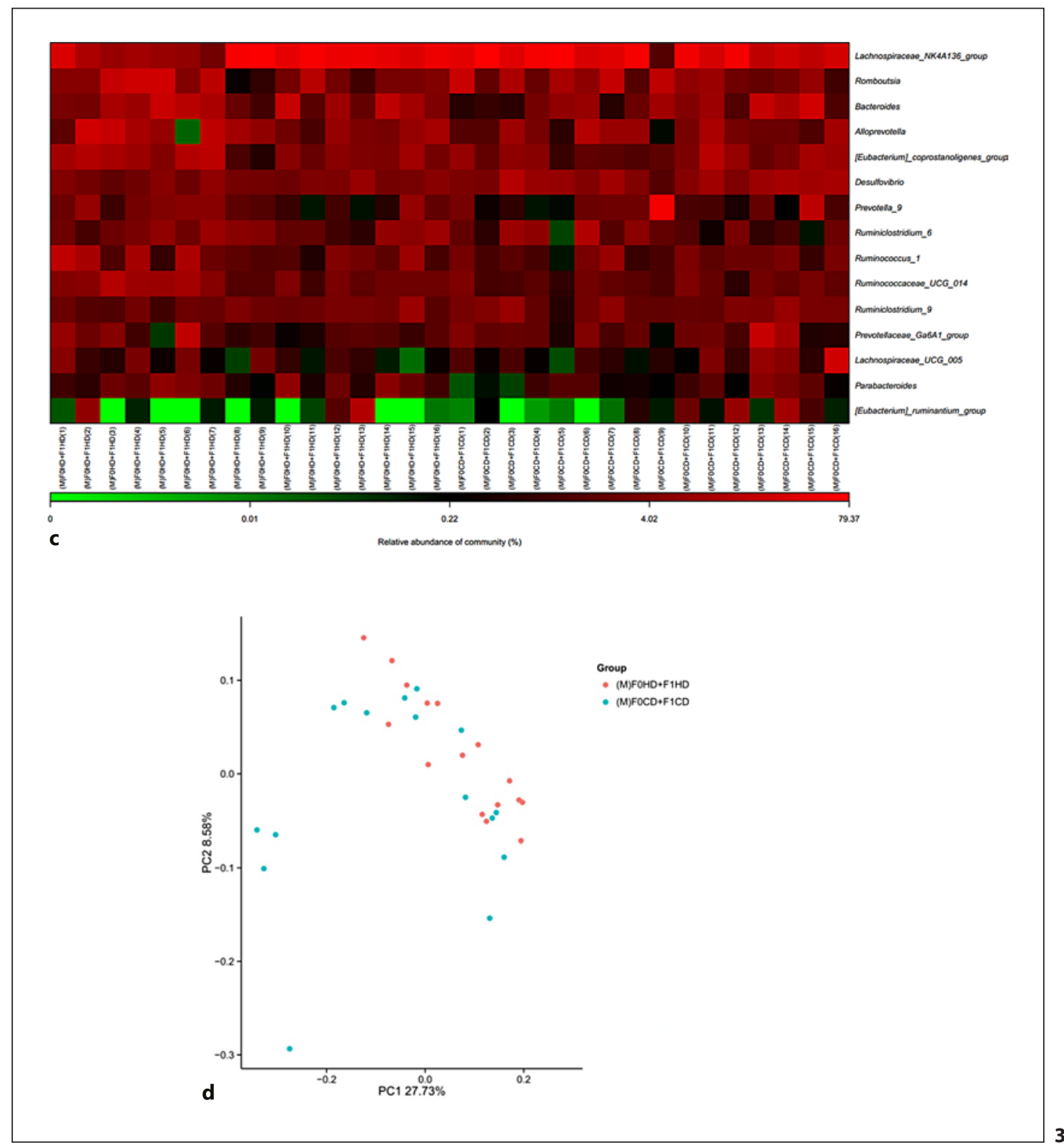

of Alloprevotella (Bacteroidetes) $\left(r^{2}=-0.642, p<0.01\right)$ was negatively associated with GLU and Ruminococcus_1 (Firmicutes) $\left(r^{2}=0.601, p<0.01\right)$ was positively associated with TG (Table 7).

In the F2 male offspring, the correlation analyses indicated that the abundance of Alloprevotella (Bacteroidetes) $\left(r^{2}=-0.478, p<0.01\right)$ and Ruminiclostridium_6 (Firmicutes) $\left(r^{2}=\right.$ $-0.529, p<0.01)$ were negatively associated with ALT activity. Ruminiclostridium_6 (Firmicutes) $\left(r^{2}=0.429, p<0.05\right)$ percentage were positively associated with GLU. The abundance of Alloprevotella (Bacteroidetes) $\left(r^{2}=0.416, p<0.05\right)$ and Ruminococcaceae_UCG_014 (Firmicutes) $\left(r^{2}=0.449, p<0.01\right)$ were positively associated with CHOL and Desulfovibrio (Proteobacteria) $\left(r^{2}=-0.393, p<0.05\right)$ was negatively associated with CHOL. The abundance of Ruminococcaceae_UCG_014 (Firmicutes) $\left(r^{2}=0.405, p<0.05\right)$ was positively associated with HDL and Desulfovibrio (Proteobacteria) $\left(r^{2}=-0.419, p<0.05\right)$ was negatively associated with HDL (Table 8). 
Table 6. Relative abundance (\%) of Firmicutes, Bacteroidetes and Proteobacteria in the F2 generation offspring of both sexes

\begin{tabular}{|c|c|c|c|c|}
\hline \multirow[t]{2}{*}{ Taxon } & \multicolumn{2}{|l|}{ Female } & \multicolumn{2}{|l|}{ Male } \\
\hline & $\begin{array}{l}\text { F0CD+F1CD } \\
(n=9)\end{array}$ & $\begin{array}{l}\text { F0HD+F1HD } \\
(n=13)\end{array}$ & $\begin{array}{l}\text { F0CD+F1CD } \\
(n=16)\end{array}$ & $\begin{array}{l}\text { F0HD+F1HD } \\
(n=16)\end{array}$ \\
\hline Firmicutes & $62.90 \pm 0.04$ & $61.89 \pm 0.02$ & $63.95 \pm 0.13$ & $62.71 \pm 0.10$ \\
\hline Bacteroidetes & $30.37 \pm 0.04$ & $32.13 \pm 0.02$ & $29.62 \pm 0.12$ & $31.28 \pm 0.09$ \\
\hline Proteobacteria & $5.29 \pm 0.04$ & $4.89 \pm 0.03$ & $5.40 \pm 0.02$ & $4.21 \pm 0.02$ \\
\hline
\end{tabular}

F0CD+F1CD, F2 offspring of F0 and F1 male founders fed a CD; F0HD+F1HD, F2 offspring of F0 and F1 male founders fed a HFSSD. Values are given as mean \pm SEM. ${ }^{*} p<0.05$ versus F0CD+F1CD, ${ }^{* *} p<0.01$ versus F0CD+F1CD.

Table 7. Correlation analysis between relative abundance (\%) of main bacterial genus and liver function/serum lipids in F2 generation female offspring

\begin{tabular}{|c|c|c|c|c|c|c|c|c|c|c|c|c|c|}
\hline & $\begin{array}{l}\text { Lachno- } \\
\text { spiraceae_ } \\
\text { NK4A136_ } \\
\text { group }\end{array}$ & $\begin{array}{l}\text { Rom- } \\
\text { boutsia }\end{array}$ & $\begin{array}{l}\text { Bacte- } \\
\text { roides }\end{array}$ & $\begin{array}{l}\text { Allo- } \\
\text { prevo- } \\
\text { tella }\end{array}$ & $\begin{array}{l}\text { [Eubac- } \\
\text { terium]_ } \\
\text { coprosta- } \\
\text { noligenes_- } \\
\text { group }\end{array}$ & $\begin{array}{l}\text { Desul- } \\
\text { fovi- } \\
\text { brio }\end{array}$ & $\begin{array}{l}\text { Pre- } \\
\text { vo- } \\
\text { tella_9 }\end{array}$ & $\begin{array}{l}\text { Rumini- } \\
\text { clostri- } \\
\text { dium_6 }\end{array}$ & $\begin{array}{l}\text { Rumino- } \\
\text { coccus_1 }\end{array}$ & $\begin{array}{l}\text { Rumino- } \\
\text { coccaceae_- } \\
\text { UCG_014 }\end{array}$ & $\begin{array}{l}\text { Rumini- } \\
\text { clostri- } \\
\text { dium_9 }\end{array}$ & $\begin{array}{l}\text { Prevotell- } \\
\text { aceae_- } \\
\text { Ga6A1_- } \\
\text { group }\end{array}$ & $\begin{array}{l}\text { Lach- } \\
\text { nospi- } \\
\text { raceae } \\
\text { UCG } \\
005\end{array}$ \\
\hline AST & $0.446^{*}$ & $-0.510^{*}$ & $-0.423^{*}$ & -0.090 & -0.104 & 0.334 & 0.273 & 0.007 & 0.161 & -0.076 & $0.525^{*}$ & -0.190 & 0.274 \\
\hline ALT & $0.553^{* *}$ & $-0.649 * *$ & $-0.584^{* *}$ & -0.057 & -0.096 & $0.441^{*}$ & 0.343 & 0.153 & 0.277 & -0.107 & $0.616^{* *}$ & 0.032 & 0.237 \\
\hline GLU & 0.141 & -0.299 & -0.191 & $-0.642^{* *}$ & -0.362 & 0.231 & 0.202 & -0.034 & 0.011 & -0.145 & 0.089 & -0.310 & 0.211 \\
\hline TG & 0.010 & 0.003 & 0.007 & -0.399 & 0.001 & 0.055 & 0.028 & 0.306 & $0.601^{* *}$ & 0.007 & 0.058 & -0.332 & 0.284 \\
\hline CHOL & 0.331 & -0.268 & -0.384 & -0.087 & -0.138 & 0.238 & -0.129 & -0.051 & 0.028 & -0.240 & 0.023 & 0.024 & -0.016 \\
\hline HDL & 0.423 & -0.356 & -0.420 & 0.063 & -0.110 & 0.189 & -0.052 & 0.031 & 0.079 & -0.202 & 0.118 & 0.080 & -0.018 \\
\hline LDL & 0.316 & -0.412 & -0.190 & -0.331 & 0.174 & 0.122 & -0.020 & -0.064 & 0.353 & -0.022 & 0.334 & -0.240 & 0.027 \\
\hline
\end{tabular}

AST, aspartate aminotransferase; ALT, alanine aminotransferase; GLU, glucose; TG, triacylglycerols; CHOL, cholesterol; HDL, high density lipoproteins; LDL, low density lipoproteins. ${ }^{*} p<0.05,{ }^{* *} p<0.01$.

Table 8. Correlation analysis between relative abundance (\%) of main bacterial genus and liver function/serum lipids in F2 generation male offspring

\begin{tabular}{|c|c|c|c|c|c|c|c|c|c|c|c|c|c|}
\hline & $\begin{array}{l}\text { Lachno- } \\
\text { spiraceae_ } \\
\text { NK4A136_ } \\
\text { group }\end{array}$ & $\begin{array}{l}\text { Rom- } \\
\text { boutsia }\end{array}$ & $\begin{array}{l}\text { Bacte- } \\
\text { roides }\end{array}$ & $\begin{array}{l}\text { Allo- } \\
\text { prevo- } \\
\text { tella }\end{array}$ & $\begin{array}{l}\text { [Eubac- } \\
\text { terium]_ } \\
\text { coprosta- } \\
\text { noligenes_ } \\
\text { group }\end{array}$ & $\begin{array}{l}\text { Desul- } \\
\text { fovi- } \\
\text { brio }\end{array}$ & $\begin{array}{l}\text { Pre- } \\
\text { vo- } \\
\text { tella_9 }\end{array}$ & $\begin{array}{l}\text { Rumini- } \\
\text { clostri- } \\
\text { dium_6 }\end{array}$ & $\begin{array}{l}\text { Rumino- } \\
\text { coccus_1 }\end{array}$ & $\begin{array}{l}\text { Rumino- } \\
\text { coccaceae_- } \\
\text { UCG_014 }\end{array}$ & $\begin{array}{l}\text { Rumini- } \\
\text { clostri- } \\
\text { dium_9 }\end{array}$ & $\begin{array}{l}\text { Prevotell- } \\
\text { aceae__ } \\
\text { Ga6A1_- } \\
\text { group }\end{array}$ & $\begin{array}{l}\text { Lach- } \\
\text { nospi- } \\
\text { raceae_ } \\
\text { UCG_ } \\
005\end{array}$ \\
\hline AST & -0.126 & 0.049 & 0.319 & -0.171 & 0.031 & -0.274 & -0.184 & -0.129 & 0.008 & 0.173 & -0.058 & -0.086 & -0.116 \\
\hline ALT & -0.032 & 0.041 & 0.294 & $-0.478^{* *}$ & -0.021 & -0.096 & -0.110 & $-0.529^{* *}$ & 0.026 & 0.130 & -0.029 & 0.012 & 0.093 \\
\hline GLU & -0.032 & -0.131 & 0.069 & 0.120 & -0.214 & -0.112 & -0.061 & 0.429* & -0.184 & -0.116 & 0.073 & -0.085 & $-0.354^{*}$ \\
\hline TG & -0.041 & 0.034 & 0.033 & 0.104 & -0.021 & -0.087 & 0.046 & 0.219 & -0.072 & 0.107 & 0.117 & -0.063 & -0.046 \\
\hline CHOL & -0.143 & 0.308 & 0.124 & $0.416^{*}$ & 0.235 & $-0.393^{*}$ & 0.183 & 0.193 & 0.205 & $0.449 * *$ & -0.176 & -0.225 & -0.091 \\
\hline HDL & -0.085 & 0.229 & 0.089 & 0.344 & 0.166 & $-0.419^{*}$ & 0.173 & 0.096 & 0.234 & $0.405^{*}$ & -0.211 & -0.263 & -0.176 \\
\hline LDL & 0.082 & -0.058 & 0.164 & 0.043 & -0.047 & -0.029 & -0.345 & -0.112 & -0.014 & -0.001 & 0.218 & -0.236 & -0.056 \\
\hline
\end{tabular}

AST, aspartate aminotransferase; ALT, alanine aminotransferase; GLU, glucose; TG, triacylglycerols; CHOL, cholesterol; HDL, high density lipoproteins; LDL, low density lipoproteins. ${ }^{*} p<0.05,{ }^{* *} p<0.01$. 


\section{Kidney \\ Blood Pressure \\ Research}

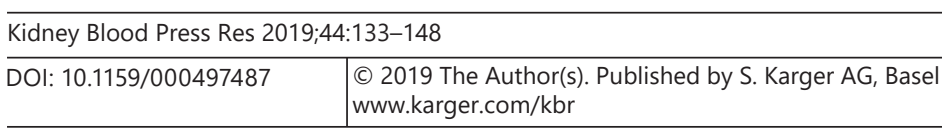

Zhang et al.: Gut Microbiome and Paternal Programming

\section{Discussion}

Most studies investigating fetal programming focused on maternal programming. So far, many different maternal programming factors have been discovered (for example over- or undernutrition [33-35], lack of micronutrients [36, 37], and stress during pregnancy [3840]). However, more recent studies showed that also paternal exposure to adverse environmental conditions can act on the offspring's phenotype in terms of fetal programming and influence later life disease risk $[11,28]$. Paternal programming has been likewise described in clinical observation studies [41,42]. So far most of the animal studies dealing with paternal programming used paternal high-fat diet models prior to mating to investigate potential underlying mechanisms. We exposed male rats to a high-salt-fat-sugar diet mimicking an unhealthy fast food diet often eaten by young men as recently described [30]. Our study was designed to examine the effect of feeding male rats HFSSD over two generations (F0 and F1) on their offspring's (F2) liver function, lipid profile and gut microbiome composition. Similar to other studies [43-45], we have shown that the F0HD+F1HD rats (two generations of paternal unhealthy diet) of both sexes had higher body weight, and higher serum aspartate aminotransferase activity at the age of 25 weeks. In addition, serum cholesterol, high density lipoproteins, low density lipoproteins were significantly higher in the male F0HD+F1HD group than in the male F0CD+F1CD group. This indicates that paternal high-salt-fat-sugar diet could lead to abnormal lipid metabolism in the later life of the offspring in a sex-specific manner. The clinical implications of these findings are unclear, since higher HDL levels are associated with a decreased risk for cardiovascular events in humans, whereas higher LDL concentrations have the opposite association with cardiovascular events.

It is important to mention the gut-liver axis, which refers to the close anatomical and functional relationship between the gastrointestinal tract and the liver [46, 47]. Such an association includes transfer of molecules associated with the gut microbiome to the liver and vice versa [48]. A growing body of evidence indicated that gut-liver axis malfunction (small intestinal bacterial overgrowth, intestinal dysbiosis, and increased intestinal permeability ["leaky gut"]) is a leading factor in the development and progression of NAFLD and obesity [49-53].

Our present study indicated that the dominant phyla of both F0CD+F1CD group and F0HD+F1HD group in the F2 offspring were Bacteroidetes, Firmicutes and Proteobacteria. These data were consistent with previous findings [54, 55]. Further analysis showed that there were significant differences in gut bacterial community composition at phyla and genus level between the F0CD+F1CD group and F0HD+F1HD group in the F2 offspring of both sexes. Of interest, our present study showed that the variations of the relative abundance (percentage) of bacterial genus of the F2 offspring were associated with liver function disorder induced by a paternal pre-conceptional unhealthy diet. Although we demonstrated correlations between liver functional parameters/lipids and the variations of the relative abundance (percentage) of bacterial genus, the causality remains to be established in further studies. Stool transplantation studies transferring stool from F2 offspring to rats with parents and grandparents on a completely normal rat diet might be a useful tool to analyze whether the observed alterations of liver function and/or lipid metabolism in the F2 offspring are causally related to alterations in the gut microbiome.

\section{Conclusion}

Our data suggest that paternal HFSSD over two generations resulted in mild liver functional alterations and intestinal microbial disorders in the F2 offspring as well as lipid profile alterations in male offspring only in later life. Our work is novel in showing the interplay 
between paternal pre-conceptional unhealthy diet, liver function alterations, lipid profile and gut microbiota. Therefore, as both gut microbiota and paternal programming become more evident and prevalent players in obesity and T2DM, a better understanding of the role of the gut microbiota in paternal programming might provide novel perspectives regarding its pathophysiological relevance and pave the way for new therapeutic principles of obesity and T2DM. Further studies should be performed to confirm these results.

\section{Acknowledgements}

This project was supported by National Natural Science Foundation of China (Grant No. 81873861) and Hunan Province Natural Science Foundation (Grant No. 2018JJ3366).

\section{Statements of Ethics}

The study was approved by the animal welfare committee of the Hunan Normal University, Changsha, China

\section{Disclosure Statement}

The authors declare that there are no competing financial interests.

\section{References}

1 Barker DJ. The fetal and infant origins of adult disease. BMJ. 1990 Nov;301(6761):1111.

2 Barker DJ. Fetal origins of coronary heart disease. BMJ. 1995 Jul;311(6998):171-4.

3 Lu YP, Reichetzeder C, Prehn C, Yin LH, Yun C, Zeng S, et al. Cord Blood Lysophosphatidylcholine 16: 1 is Positively Associated with Birth Weight. Cell Physiol Biochem. 2018;45(2):614-24.

4 Arumugam M, Raes J, Pelletier E, Le Paslier D, Yamada T, Mende DR, et al.; MetaHIT Consortium. Enterotypes of the human gut microbiome. Nature. 2011 May;473(7346):174-80.

5 Human Microbiome Project Consortium. Structure, function and diversity of the healthy human microbiome. Nature. 2012 Jun;486(7402):207-14.

6 Human Microbiome Project Consortium. A framework for human microbiome research. Nature. 2012 Jun; 486(7402):215-21.

7 Turnbaugh PJ, Hamady M, Yatsunenko T, Cantarel BL, Duncan A, Ley RE, et al. A core gut microbiome in obese and lean twins. Nature. 2009 Jan;457(7228):480-4.

8 Ottman N, Smidt H, de Vos WM, Belzer C. The function of our microbiota: who is out there and what do they do? Front Cell Infect Microbiol. 2012 Aug;2:104.

9 Tilg H, Moschen AR. Microbiota and diabetes: an evolving relationship. Gut. 2014 Sep;63(9):1513-21.

10 Barker DJ. The developmental origins of adult disease. J Am Coll Nutr. 2004 Dec;23(6 Suppl):588S-95S.

11 Reichetzeder C, Dwi Putra SE, Li J, Hocher B. Developmental Origins of Disease - Crisis Precipitates Change. Cell Physiol Biochem. 2016;39(3):919-38.

12 David LA, Maurice CF, Carmody RN, Gootenberg DB, Button JE, Wolfe BE, et al. Diet rapidly and reproducibly alters the human gut microbiome. Nature. 2014 Jan;505(7484):559-63.

13 Ma J, Prince AL, Bader D, Hu M, Ganu R, Baquero K, et al. High-fat maternal diet during pregnancy persistently alters the offspring microbiome in a primate model. Nat Commun. 2014 May;5(1):3889.

14 Sonnenburg ED, Smits SA, Tikhonov M, Higginbottom SK, Wingreen NS, Sonnenburg JL. Diet-induced extinctions in the gut microbiota compound over generations. Nature. 2016 Jan;529(7585):212-5.

15 Collado MC, Isolauri E, Laitinen K, Salminen S. Distinct composition of gut microbiota during pregnancy in overweight and normal-weight women. Am J Clin Nutr. 2008 Oct;88(4):894-9.

16 Collado MC, Isolauri E, Laitinen K, Salminen S. Effect of mother's weight on infant's microbiota acquisition, composition, and activity during early infancy: a prospective follow-up study initiated in early pregnancy. Am J Clin Nutr. 2010 Nov;92(5):1023-30.

17 Santacruz A, Collado MC, García-Valdés L, Segura MT, Martín-Lagos JA, Anjos T, et al. Gut microbiota composition is associated with body weight, weight gain and biochemical parameters in pregnant women. Br J Nutr. 2010 Jul;104(1):83-92. 


\begin{tabular}{l|l}
\hline Kidney Blood Press Res 2019;44:133-148 \\
\hline DOI: 10.1159/000497487 & $\begin{array}{l}\text { @ 2019 The Author(s). Published by S. Karger AG, Basel } \\
\text { www.karger.com/kbr }\end{array}$ \\
\hline
\end{tabular}

Zhang et al.: Gut Microbiome and Paternal Programming

18 Paul HA, Bomhof MR, Vogel HJ, Reimer RA. Diet-induced changes in maternal gut microbiota and metabolomic profiles influence programming of offspring obesity risk in rats. Sci Rep. 2016 Feb;6(1):20683.

19 Charbonneau MR, O’Donnell D, Blanton LV, Totten SM, Davis JC, Barratt MJ, et al. Sialylated Milk Oligosaccharides Promote Microbiota-Dependent Growth in Models of Infant Undernutrition. Cell. 2016 Feb;164(5):85971.

20 Blanton LV, Charbonneau MR, Salih T, Barratt MJ, Venkatesh S, Ilkaveya O, et al. Gut bacteria that prevent growth impairments transmitted by microbiota from malnourished children. Science. 2016 Feb;351(6275): 351.

21 Hooper LV, Littman DR, Macpherson AJ. Interactions between the microbiota and the immune system. Science. 2012 Jun;336(6086):1268-73.

22 Olszak T, An D, Zeissig S, Vera MP, Richter J, Franke A, et al. Microbial exposure during early life has persistent effects on natural killer T cell function. Science. 2012 Apr;336(6080):489-93.

23 Round JL, Mazmanian SK. The gut microbiota shapes intestinal immune responses during health and disease. Nat Rev Immunol. 2009 May;9(5):313-23.

24 Clarke G, Grenham S, Scully P, Fitzgerald P, Moloney RD, Shanahan F, et al. The microbiome-gut-brain axis during early life regulates the hippocampal serotonergic system in a sex-dependent manner. Mol Psychiatry. 2013 Jun;18(6):666-73.

25 Foster JA, McVey Neufeld KA. Gut-brain axis: how the microbiome influences anxiety and depression. Trends Neurosci. 2013 May;36(5):305-12.

26 Boursier J, Mueller O, Barret M, Machado M, Fizanne L, Araujo-Perez F, et al. The severity of nonalcoholic fatty liver disease is associated with gut dysbiosis and shift in the metabolic function of the gut microbiota. Hepatology. 2016 Mar;63(3):764-75.

27 McPherson NO, Fullston T, Aitken RJ, Lane M. Paternal obesity, interventions, and mechanistic pathways to impaired health in offspring. Ann Nutr Metab. 2014;64(3-4):231-8.

28 Li J, Tsuprykov O, Yang X, Hocher B. Paternal programming of offspring cardiometabolic diseases in later life. J Hypertens. 2016 Nov;34(11):2111-26.

29 Chen YP, Xiao XM, Li J, Reichetzeder C, Wang ZN, Hocher B. Paternal body mass index (BMI) is associated with offspring intrauterine growth in a gender dependent manner. PLoS One. 2012;7(5):e36329.

30 Li J, Lu YP, Tsuprykov O, Hasan AA, Reichetzeder C, Tian M, et al. Folate treatment of pregnant rat dams abolishes metabolic effects in female offspring induced by a paternal pre-conception unhealthy diet. Diabetologia. 2018 Aug;61(8):1862-76.

31 Ng SF, Lin RC, Laybutt DR, Barres R, Owens JA, Morris MJ. Chronic high-fat diet in fathers programs $\beta$-cell dysfunction in female rat offspring. Nature. 2010 0ct;467(7318):963-6.

32 Hocher B, Adamski J. Metabolomics for clinical use and research in chronic kidney disease. Nat Rev Nephrol. 2017 May;13(5):269-84.

33 Rodríguez-González GL, Vega CC, Boeck L, Vázquez M, Bautista CJ, Reyes-Castro LA, et al. Maternal obesity and overnutrition increase oxidative stress in male rat offspring reproductive system and decrease fertility. Int J Obes. 2015 Apr;39(4):549-56.

34 Latouche C, Heywood SE, Henry SL, Ziemann M, Lazarus R, El-Osta A, et al. Maternal overnutrition programs changes in the expression of skeletal muscle genes that are associated with insulin resistance and defects of oxidative phosphorylation in adult male rat offspring. J Nutr. 2014 Mar;144(3):237-44.

35 Li M, Reynolds CM, Gray C, Vickers MH. Preweaning GH Treatment Normalizes Body Growth Trajectory and Reverses Metabolic Dysregulation in Adult Offspring After Maternal Undernutrition. Endocrinology. 2015 Sep;156(9):3228-38.

36 Reichetzeder C, Chen H, Föller M, Slowinski T, Li J, Chen YP, et al. Maternal vitamin D deficiency and fetal programming—lessons learned from humans and mice. Kidney Blood Press Res. 2014;39(4):315-29.

37 Roy S, Sable P, Khaire A, Randhir K, Kale A, Joshi S. Effect of maternal micronutrients (folic acid and vitamin B12) and omega 3 fatty acids on indices of brain oxidative stress in the offspring. Brain Dev. 2014 Mar;36(3): 219-27.

38 Brunton PJ. Effects of maternal exposure to social stress during pregnancy: consequences for mother and offspring. Reproduction. 2013 Oct;146(5):R175-89.

39 Betts KS, Williams GM, Najman JM, Alati R. The relationship between maternal depressive, anxious, and stress symptoms during pregnancy and adult offspring behavioral and emotional problems. Depress Anxiety. 2015 Feb;32(2):82-90.

40 Li J, Wang ZN, Chen YP, Dong YP, Shuai HL, Xiao XM, et al. Late gestational maternal serum cortisol is inversely associated with fetal brain growth. Neurosci Biobehav Rev. 2012 Mar;36(3):1085-92.

41 Yen AM, Boucher BJ, Chiu SY, Fann JC, Chen SL, Huang KC, et al. Longer Duration and Earlier Age of Onset of Paternal Betel Chewing and Smoking Increase Metabolic Syndrome Risk in Human Offspring, Independently, in a Community-Based Screening Program in Taiwan. Circulation. 2016 Aug;134(5):392-404.

42 Carslake D, Pinger PR, Romundstad P, Davey Smith G. Early-Onset Paternal Smoking and Offspring Adiposity: Further Investigation of a Potential Intergenerational Effect Using the HUNT Study. PLoS One. 2016 Dec; 11(12):e0166952.

43 Page KC, Malik RE, Ripple JA, Anday EK. Maternal and postweaning diet interaction alters hypothalamic gene expression and modulates response to a high-fat diet in male offspring. Am J Physiol Regul Integr Comp Physiol. 2009 Oct;297(4):R1049-57. 
44 Bruce KD, Cagampang FR, Argenton M, Zhang J, Ethirajan PL, Burdge GC, et al. Maternal high-fat feeding primes steatohepatitis in adult mice offspring, involving mitochondrial dysfunction and altered lipogenesis gene expression. Hepatology. 2009 Dec;50(6):1796-808.

45 Elahi MM, Cagampang FR, Mukhtar D, Anthony FW, Ohri SK, Hanson MA. Long-term maternal high-fat feeding from weaning through pregnancy and lactation predisposes offspring to hypertension, raised plasma lipids and fatty liver in mice. Br J Nutr. 2009 Aug;102(4):514-9.

46 Minemura M, Shimizu Y. Gut microbiota and liver diseases. World J Gastroenterol. 2015 Feb;21(6):1691-702.

47 Usami M, Miyoshi M, Yamashita H. Gut microbiota and host metabolism in liver cirrhosis. World J Gastroenterol. 2015 Nov;21(41):11597-608.

48 Sharma V, Garg S, Aggarwal S. Probiotics and liver disease. Perm J. 2013;17(4):62-7.

49 Le Roy T, Llopis M, Lepage P, Bruneau A, Rabot S, Bevilacqua C, et al. Intestinal microbiota determines development of non-alcoholic fatty liver disease in mice. Gut. 2013 Dec;62(12):1787-94.

50 Paolella G, Mandato C, Pierri L, Poeta M, Di Stasi M, Vajro P. Gut-liver axis and probiotics: their role in nonalcoholic fatty liver disease. World J Gastroenterol. 2014 Nov;20(42):15518-31.

51 Vajro P, Paolella G, Fasano A. Microbiota and gut-liver axis: their influences on obesity and obesity-related liver disease. J Pediatr Gastroenterol Nutr. 2013 May;56(5):461-8.

52 Miele L, Marrone G, Lauritano C, Cefalo C, Gasbarrini A, Day C, et al. Gut-liver axis and microbiota in NAFLD: insight pathophysiology for novel therapeutic target. Curr Pharm Des. 2013;19(29):5314-24.

53 Hocher B, Haumann H, Rahnenführer J, Reichetzeder C, Kalk P, Pfab T, et al. Maternal eNOS deficiency determines a fatty liver phenotype of the offspring in a sex dependent manner. Epigenetics. 2016 Jul;11(7):539-52.

54 Sun H, Wang N, Cang Z, Zhu C, Zhao L, Nie X, et al. Modulation of Microbiota-Gut-Brain Axis by Berberine Resulting in Improved Metabolic Status in High-Fat Diet-Fed Rats. Obes Facts. 2016;9(6):365-78.

55 Zheng J, Xiao X, Zhang Q, Yu M, Xu J, Qi C, et al. The effects of maternal and post-weaning diet interaction on glucose metabolism and gut microbiota in male mice offspring. Biosci Rep. 2016 Jun;36(3):e00341-00341. 